\title{
Analysis and Design of a High-Efficiency Boundary Conduction Mode Tapped-Inductor Boost LED Driver for Mobile Products
}

\author{
Jeong-il Kang ${ }^{\dagger}$, Sang-Kyoo Han*, and Jonghee Han ${ }^{* *}$ \\ $\dagger^{* * *}$ Research \& Development Office, Visual Display, Samsung Electronics Co., Ltd., Suwon, Korea \\ "School of Electrical Engineering, College of EECS, Kookmin University, Seoul, Korea
}

\begin{abstract}
For low-power high-frequency LED driver applications in small form factor mobile products, a high-efficiency boundary conduction mode tapped-inductor boost converter is proposed. In the proposed converter, the switch and the diode achieve soft-switching, the diode reverse-recovery is alleviated, and the switching frequency is very insensitive to output voltage variations. The circuit is quantitatively characterized, and the design guidelines are presented. Experimental results from an LED backlight driver prototype for a 14 inch notebook computer are also presented.
\end{abstract}

Key words: Current control, DC-DC power conversion, Efficiency, ZVS converters

\section{INTRODUCTION}

Liquid crystal displays (LCD) are currently in a very wide use as flat panel display devices, whose diagonal length ranges from sub-inch to more than 100 inches. Since LCDs cannot generate light by themselves, they need an illumination unit to make an image visible. This illumination unit is often called a backlight unit, and light emitting diodes (LED) are recently achieving a dominant position as this light source since they have many excellent features such as high energy efficiency, high scalability, long lifetime, and eco-friendliness when compared to conventional cold-cathode fluorescent lamps [1]-[5].

In general, a smaller backlight unit tends to have a smaller number of LEDs, as shown in Fig. 1 (a). However, this is not always true since design requirements are becoming more stringent and require a narrower bezel. Due to the point-source nature of LEDs, narrow-bezel products require a significantly larger number of LEDs than wide-bezel products to maintain

Manuscript received Dec. 10, 2013; accepted Apr. 18, 2014

Recommended for publication by Associate Editor Joung-Hu Park.

${ }^{\dagger}$ Corresponding Author: jeongil.kang@samsung.com

Tel: +82-31-277-2984, Samsung Electronics Co., Ltd.

* School of Electrical Engineering, College of EECS, Kookmin University, Korea

${ }^{* *}$ Research \& Development Office, Visual Display, Samsung Electronics Co., Ltd., Korea good brightness uniformity for the same display area. This is shown in Fig. 1 (b). Even a small-sized backlight unit whose diagonal length is only a few inches can be easily found to have as many as several tens of LEDs. In addition, the voltage required to drive them often exceeds $50 \mathrm{~V}$.

The conventional boost converter shown in Fig. 2 is widely being used as, or as a part of, constant current LED drivers for the backlight units where the required output voltage is higher than the available input voltages to the circuit. It is preferable for this converter to operate in continuous conduction mode (CCM) to minimize the conduction loss caused by a high input current ripple [6], [7]. However, in low power applications such as the LED backlight drivers for small form factor mobile products, the conduction loss is not that critical and often considered to be insignificant since the input current is relatively small. In such cases, operation in boundary conduction mode (BCM), which is also known as critical conduction mode (CRM), finds a good opportunity since it features no diode reverse-recovery problem, no right half-plane zero, and no need for slope compensation. Furthermore, the switch and the diode can respectively achieve zero voltage switching (ZVS) and zero current switching (ZCS) through the resonance of the boost inductor and the output capacitance of switching devices if appropriate operating conditions are provided [6]-[15].

The biggest problem with the BCM boost converter for LED 


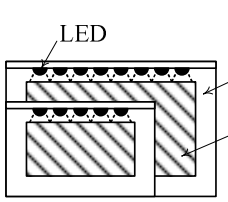

(a) different display sizes.

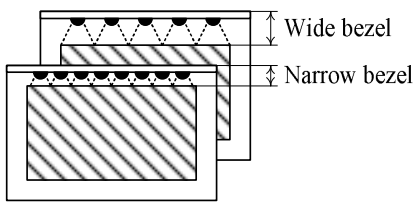

(b) different bezel widths.
Fig. 1. Typical LED backlight unit.

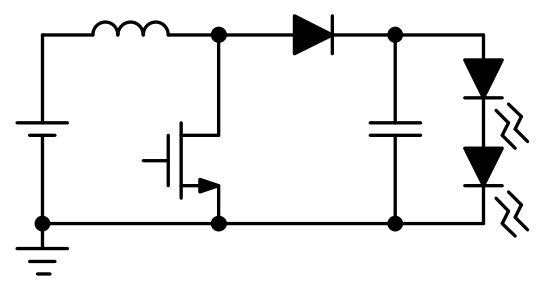

Fig. 2. Conventional boost LED driver.

driver applications is that even a small variation in the output voltage causes a significant change in the switching frequency. Unfortunately, the voltage across the LED is given as a ranged value due to its dependencies on the temperature, the material used, the manufacturer, the production lot, and so on [3]-[5]. To make matters worse, the LED voltage can change even beyond its specified normal range due to changes in the material properties through long-term aging. Consequently, the $\mathrm{BCM}$ boost LED driver needs to manage wide variations in the switching frequency, which makes it difficult to optimize the circuit from the standpoint of efficiency, transient response, ripple content, electromagnetic interference (EMI), and so on. This, in spite of its many attractive features, restricts the use of the BCM boost converter as an LED driver.

As an alternative to the conventional $\mathrm{BCM}$ boost converter, a BCM tapped-inductor boost (TIB) converter for LED driver applications is proposed in this paper. When compared to the conventional BCM boost converter, the proposed converter shows a switching frequency which is very insensitive to changes in the output voltage [15]. It also shows a higher step-up ratio and a lower switch voltage stress than its conventional counterpart [16]-[18]. Moreover, the proposed converter provides ZVS of the switch and ZCS of the diode, which allows for a higher-frequency operation with a more compact circuit implementation. Therefore, the proposed BCM TIB converter is considered to be a good candidate for constant current LED driver applications especially for small form factor mobile products. In this paper, the steady-state and soft-switching operations of the proposed converter are analyzed, various voltage and current stresses are quantitatively characterized, and a design guideline optimizing the tapped-inductor loss is presented. Experimental results from a prototype driver circuit for a 14 inch LED backlight for a notebook computer are also presented.

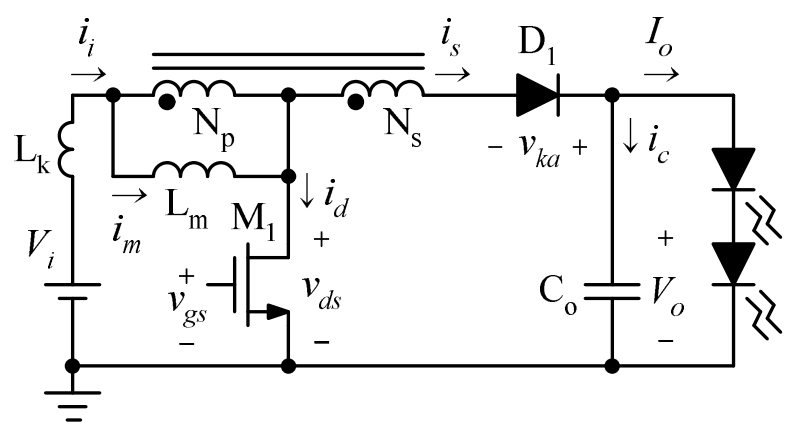

Fig. 3. Tapped-inductor boost (TIB) topology.

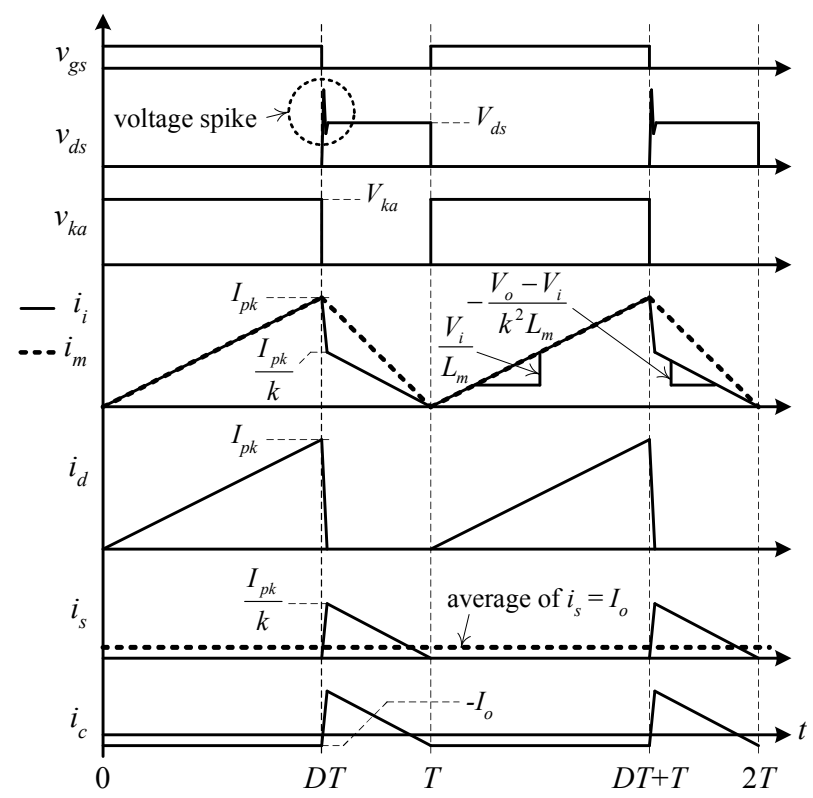

Fig. 4. Key operating waveforms.

\section{ANALYSIS OF THE BCM TIB LED DRIVER}

A schematic diagram and the key operating waveforms of the proposed BCM TIB converter are shown in Fig. 3 and Fig. 4 , respectively. Switch $M_{1}$ operates at a frequency of $f=1 / T$ and a duty ratio of $D$. The tapped-inductor is modeled as an ideal transformer with a turns ratio of $N=N_{s} / N_{p}$, a magnetizing inductance $L_{m}$, which is referred to the primary side, and a leakage inductance $L_{k}$, which is referred to the primary side. The value of $L_{m}$ is assumed to be much larger than that of $L_{k}$. The input voltage $V_{i}$, the output voltage $V_{o}$, and the output current $I_{o}$ are assumed to be ripple-free and constant. For convenience in developing equations, $1+N$ is denoted by $k$.

\section{A. Steady-state Analysis}

The topological stages according to the switch state are shown in Fig. 5. Initially, switch $M_{1}$ is assumed to be in the off-state. It is turned on as soon as the decreasing magnetizing current $i_{m}$ reached zero at $t=0$. 
On-mode [0 DT]: This mode begins when switch $M_{1}$ turns on. Since the output diode $D_{1}$ is reverse-biased, there is no induced current in the coupled windings, and all of the input current, denoted by $i_{i}$, flows through $L_{k}$ and $L_{m}$. From the assumption that $L_{m} \gg L_{k}$, the leakage inductance $L_{k}$ in the input current loop can be neglected, and the voltage across $L_{m}$ is equal to $V_{i}$. The magnetizing current $i_{m}$, which is equal to $i_{i}$, increases linearly from zero to $I_{p k}$.

The voltage stress on $D_{1}$, shown by $V_{k a}$ in Fig. 4 , is equal to the sum of $V_{o}$ and $V_{i}$ reflected to the secondary side. It can be normalized with respect to $V_{i}$ as:

$$
V_{k a} / V_{i}=V_{o} / V_{i}+N
$$

Off-mode $[\boldsymbol{D T} \sim \boldsymbol{T}]$ : This mode begins when switch $M_{1}$ turns off at $t=D T$. Due to the leakage inductance $L_{k}$, the switch current cannot instantaneously fall to zero the moment $M_{1}$ turns off. Therefore, a high voltage spike can be developed at the switch drain, as illustrated in Fig. 4. To alleviate this, the tapped-inductor windings need to be tightly coupled, or a snubber circuit should be considered.

After a brief transient due to $L_{k}$, switch $M_{1}$ enters the off-state, and diode $D_{1}$ enters the on-state, as shown in Fig. 5(b). The input current $i_{i}$ is equal to the secondary current $i_{s}$, and the magnetizing current $i_{m}$ is equal to sum of the input current $i_{i}$ and the primary induced current of $N \cdot i_{s}$, resulting in $i_{m}=k \cdot i_{i}$. The input current $i_{i}$ decreases linearly from $I_{p k} / k$ to zero during this mode as the magnetizing current $i_{m}$ decreases linearly from $I_{p k}$ to zero. Since the equivalent inductance of the tapped-inductor in this stage is $L_{k}+k^{2} L_{m}$ [16], the leakage inductance $L_{k}$, which is assumed to be much smaller than $k^{2} L_{m}$, can be safely neglected.

The winding voltages are proportional to their number of turns. Therefore, the voltage across $L_{m}$ can be obtained to be $\left(V_{i}-V_{o}\right) / k$. Subtracting this voltage from $V_{i}$ gives the voltage stress on $M_{1}$, shown by $V_{d s}$ in Fig. 4, which can be normalized with respect to $V_{i}$ as:

$$
V_{d s} / V_{i}=\left(V_{o} / V_{i}+N\right) / k
$$

This mode ends when the input current reaches zero and the tapped-inductor is demagnetized.

Neglecting the effect of $L_{k}$, whose contribution to the step-up ratio is very small, the step-up ratio of the BCM TIB converter and the inverse function can be obtained by applying the volt-second balance to $L_{m}$ for one switching cycle as:

$$
\frac{V_{o}}{V_{i}}=\frac{1+N D}{1-D} \rightarrow D=\frac{V_{o} / V_{i}-1}{V_{o} / V_{i}+N}
$$

which is identical to the CCM TIB converter.

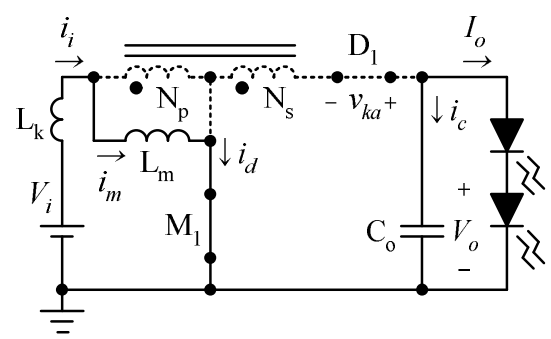

(a) switch ON

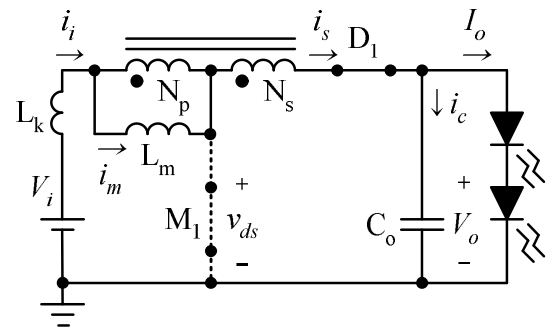

(b) switch OFF.

Fig. 5. Topological stages.

Since the average current in the output capacitor $C_{o}$ is zero in the steady-state, the relationship between $I_{p k}$ and $I_{o}$ can be obtained by averaging $i_{s}$ for one switching cycle to equate with $I_{o}$ as:

$$
I_{p k} / I_{o}=2\left(V_{o} / V_{i}+N\right),
$$

where $D$ is eliminated using (3).

The curves of $D, I_{p k} / I_{o}, V_{d s} / V_{i}$, and $V_{k a} / V_{i}$, given by (3), (4), (2), and (1) respectively, are plotted in Fig. 6 as functions of $V_{o} / V_{i}$ for different values of $N$. Since the proposed converter is supposed to supply a constant current to an LED string whose forward voltage is given as a ranged value, it is reasonable to study the characteristics as functions of $V_{o} / V_{i}$ rather than as functions of the duty ratio.

Fig. 6 and the corresponding equations show that decrements of $D$ and $V_{d s} / V_{i}$, over an increment of $N$ at a given $V_{o} / V_{i}$, decrease as $N$ increases. Therefore, $D$ and $V_{d s} / V_{i}$ converge to 0 and 1 respectively when $N$ keeps increasing. On the other hand, increments of $I_{p k} / I_{o}$ and $V_{k a} / V_{i}$, over an increment of $N$ at a given $V_{o} / V_{i}$, do not vanish as $N$ increases. Therefore, $I_{p k} / I_{o}$ and $V_{k a} / V_{i}$ consistently increase as $N$ increases. This means that the value of $N$ should not be too large. If it is, the disadvantages from the increased $I_{p k}$ and $V_{k a}$ overtake the advantages from the decreased $D$ and $V_{d s}$.

The switching frequency $f$ of the BCM TIB converter can be obtained by equating $I_{p k}$, given by (4), with the increase in $i_{m}$ during On-mode as:

$$
f=\frac{V_{i}\left(V_{o} / V_{i}-1\right)}{2 L_{m} I_{o}\left(V_{o} / V_{i}+N\right)^{2}},
$$



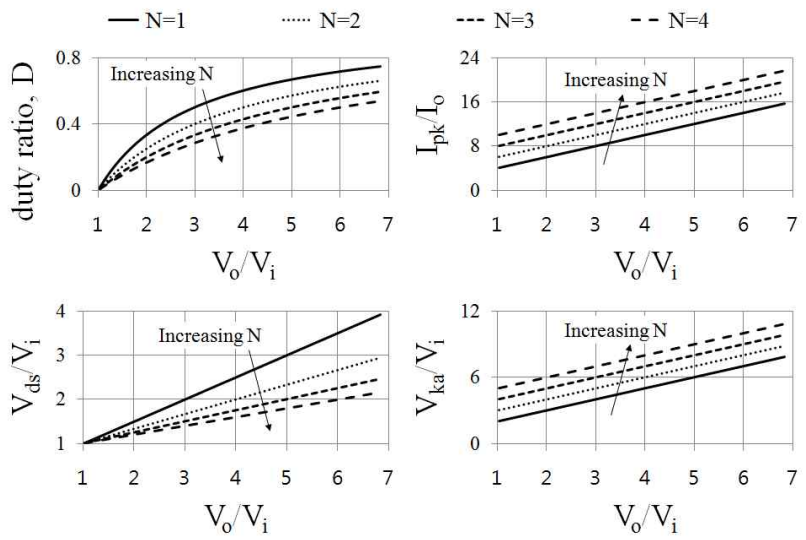

Fig. 6. Tendencies of key values to $V_{o} / V_{i}$ variation.

where $D$ is eliminated using (3).

For the best visualization of (5), the operating conditions are exemplified as $V_{i}=14 \mathrm{~V}, V_{o}=55 \mathrm{~V}, I_{o}=44 \mathrm{~mA}$, and $f=220 \mathrm{kHz}$. The values of $L_{m}$ satisfying these conditions are determined using (5) for different values of $N$. Then, the switching frequencies of the proposed converter for different pairs of $N$ and $L_{m}$ are calculated from (5) as functions of $V_{o}$. The results are plotted in Fig. 7. The curve for the conventional BCM boost converter, shown by a dash-dot line, is obtained from (5) where $N=0$. It is obvious that the conventional BCM boost converter has a steep negative slope at the operating point, whereas the proposed BCM TIB converter has a much more gradual slope with a positive-going tendency as $N$ increases. This characteristic makes the proposed converter very attractive for LED driver applications since the switching frequency dependent aspects such as the efficiency, the ripple content, the EMI, and the transient response can be made very stable.

By differentiating (5) with respect to $V_{o}$ and equating the result with zero, the stationary point of (5), where the frequency change due to a small perturbation in $V_{o}$ is zero, can be found at:

$$
V_{o}=(N+2) V_{i} .
$$

\section{B. Soft-switching analysis}

Due to the BCM operation, the output diode $D_{1}$ in the Off-mode is turned off not by a change in the voltage across it but by the current gradually decreasing to zero, as shown in Fig. 4. As a result, the ZCS operation of $D_{1}$ is achieved, and the reverse-recovery current of $D_{1}$ is alleviated.

The moment $D_{1}$ turns off, all of the currents $i_{i}, i_{m}$, and $i_{s}$ reach zero, and the output capacitance of the switching devices begins to resonate with the magnetizing inductance. The resonant elements are identified by dashed rectangles in Fig. 8, where the leakage inductance $L_{k}$ is again neglected since the resonant behavior is governed by $L_{m}$ which is much larger than $L_{k}$.

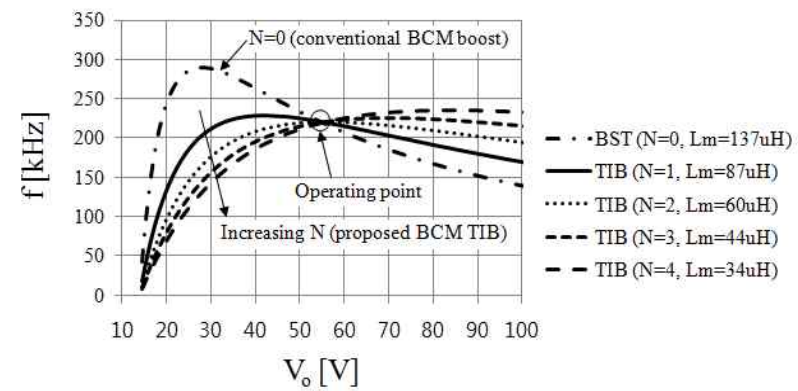

Fig. 7. Tendency of switching frequency to $V_{o}$ variation.

The resonant waveforms of $v_{d s}, i_{i}$, and $v_{k a}$ are illustrated in Fig. 9. The input current $i_{i}$ is assumed to reach zero at $t=t_{z c 1}$, and $M_{1}$ is assumed to turn on at $t=t_{z c 2}$, the moment $i_{i}$ crosses zero to change its direction. The solid line shows a case where the ZVS of the switch can be provided since the resonant amplitude of $v_{d s}$ is large enough to touch zero at $t=t_{z v}$. In this case, the voltage $v_{d s}$ cannot decrease further below zero after $t=t_{z v}$ since the body diode of $M_{1}$ turns on. If $M_{1}$ turns on at any time between $t=t_{z v}$ and $t=t_{z c 2}$ while the voltage $v_{d s}$ is clamped at zero, the ZVS of $M_{1}$ can be achieved. The dashed lines in Fig. 9 illustrate the opposite case where the voltage $v_{d s}$ cannot reach zero, and the ZVS of $M_{1}$ cannot be achieved.

To derive the ZVS condition for $M_{1}$, a set of differential equations are set up for the resonant elements as:

$$
\begin{gathered}
v_{m}=L_{m} \frac{d i_{m}}{d t}=L_{m} \frac{d i_{d}}{d t}+k L_{m} \frac{d i_{s}}{d t} \\
i_{d}=C_{d s} \frac{d v_{d s}}{d t} \\
i_{s}=-C_{k a} \frac{d v_{k a}}{d t}=-C_{k a}\left(\frac{d V_{o}}{d t}+N \frac{d v_{m}}{d t}-\frac{d v_{d s}}{d t}\right) \\
=-N C_{k a} \frac{d v_{m}}{d t}+C_{k a} \frac{d v_{d s}}{d t} .
\end{gathered}
$$

Substituting (8) and (9) into (7) results in:

$$
v_{m}=L_{m}\left(C_{d s}+k C_{k a}\right) \frac{d^{2} v_{d s}}{d t^{2}}-k L_{m} N C_{k a} \frac{d^{2} v_{m}}{d t^{2}} .
$$

Substituting $v_{m}$ into (10) with $V_{i}-v_{d s}$ results in a second order differential equation for $v_{d s}$ as:

$$
L_{m}\left(C_{d s}+k^{2} C_{k a}\right) \frac{d^{2} v_{d s}}{d t^{2}}+v_{d s}=V_{i}
$$

where $v_{d s}(0)=\left(V_{o}+N V_{i}\right) / k$. By solving (11), the equation for $v_{d s}$ can be obtained as:

$$
v_{d s}\left(t-t_{z c 1}\right)=V_{i}+\frac{V_{o}-V_{i}}{k} \cos \omega\left(t-t_{z c 1}\right),
$$




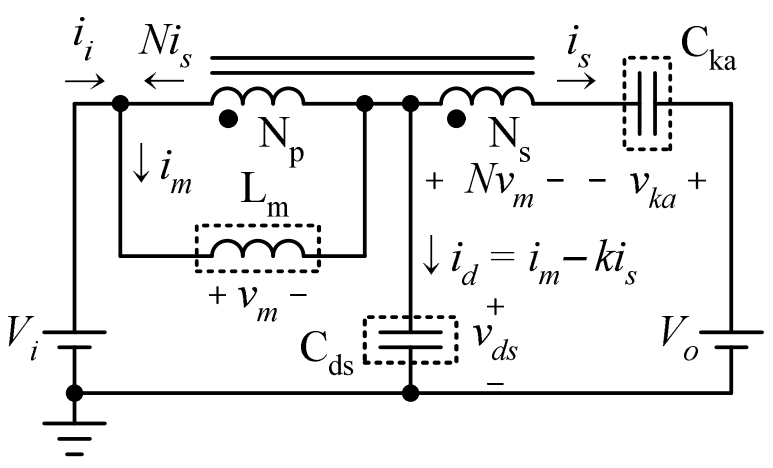

Fig. 8. Resonant circuit after tapped-inductor demagnetization.

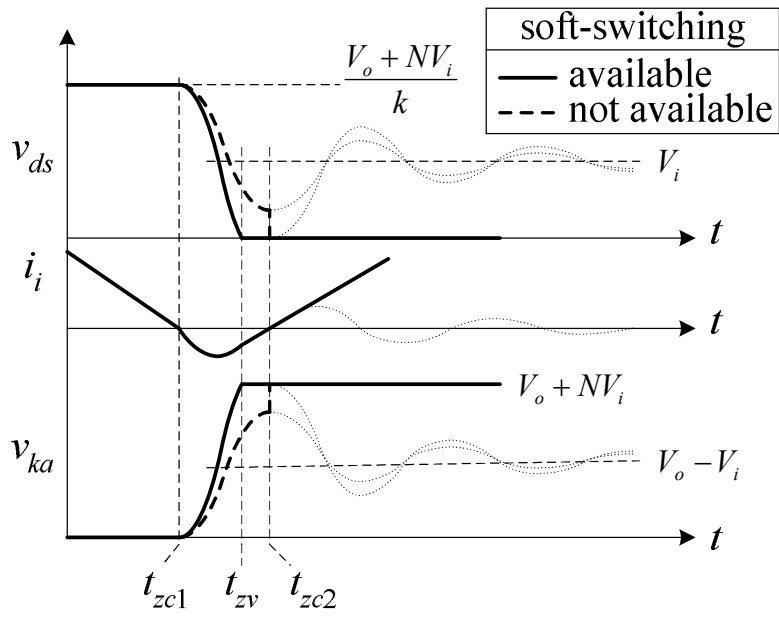

Fig. 9. Waveforms after tapped-inductor demagnetization.

where $\omega=\left\{L_{m}\left(C_{d s}+k^{2} C_{k a}\right)\right\}^{-1 / 2}$. Since $v_{k a}$ is equal to $V_{o}+N v_{m}-v_{d s}$ and $v_{m}$ is equal to $V_{i}-v_{d s}$, the equation for $v_{k a}$ can be obtained as:

$$
v_{k a}\left(t-t_{z c 1}\right)=\left(V_{o}-V_{i}\right)\left\{1-\cos \omega\left(t-t_{z c 1}\right)\right\} .
$$

To provide the ZVS of $M_{1}$, the minimum value of (12) should be negative, which results in the ZVS condition for $M_{1}$ as:

$$
N<V_{o} / V_{i}-2 \rightarrow D>0.5
$$

where $V_{o} / V_{i}$ is eliminated by using (3).

It should be noted that $v_{k a}$ stays at $V_{o}+N V_{i}$ while $v_{d s}$ is clamped at zero. Since this voltage is equal to the diode turn off voltage given by (1), a fast $d v_{k a} / d t$ associated problems such as the EMI due to the spiky charging current of $C_{k a}$ at the moment of the switch turn-on is also mitigated if (14) is satisfied.

\section{Current stress analysis}

Since the switching losses in the proposed converter are very small due to the soft-switching operation, the conduction loss
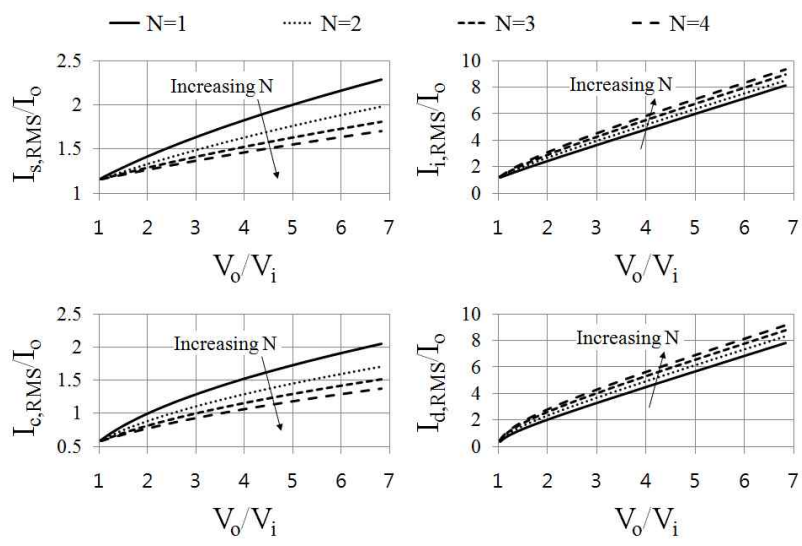

Fig. 10. Trend of normalized RMS currents to $V_{o} / V_{i}$ variation.

due to the parasitic resistance in the circuit is the next one to be considered. Neglecting the effect of $L_{k}$, the major RMS current stresses normalized with respect to $I_{o}$ can be calculated from the waveforms in Fig. 4 as:

$$
\begin{aligned}
& \frac{I_{i, R M S}}{I_{o}}=\frac{2}{\sqrt{3}} \frac{\sqrt{1+D N(N+2)}}{1-D}, \\
& \frac{I_{d, R M S}}{I_{o}}=\frac{2}{\sqrt{3}} \frac{(1+N) \sqrt{D}}{1-D}, \\
& \frac{I_{s, R M S}}{I_{o}}=\frac{2}{\sqrt{3}} \frac{1}{\sqrt{1-D}}, \\
& \frac{I_{c, R M S}}{I_{o}}=\frac{1}{\sqrt{3}} \frac{\sqrt{1+3 D}}{\sqrt{1-D}} .
\end{aligned}
$$

By eliminating $D$ in (15)-(18) using (3), the curves of $I_{i, R M S} / I_{o}$, $I_{d, R M S} / I_{o}, I_{s, R M S} / I_{o}$, and $I_{c, R M S} / I_{o}$ are plotted in Fig. 10 as functions of $V_{o} / V_{i}$ for different values of $N$. Since the duty ratio $D$ given by (3) approaches zero as $N$ increases at a given $V_{o} / V_{i}$, both $I_{S, R M S} / I_{o}$ and $I_{c, R M S} / I_{o}$ finally converge to constant values defined by $D=0$ when $N$ keeps increasing. On the other hand, the increments of $I_{i, R M S} / I_{o}$ and $I_{d, R M S} / I_{o}$ over an increment of $N$ at a given $V_{o} / V_{i}$ do not disappear as $N$ increases. Therefore, $I_{i, R M S} / I_{o}$ and $I_{d, R M S} / I_{o}$ consistently increase as $N$ increases. This means that, if the value of $N$ is too large, the disadvantages from the increased $I_{i, R M S}$ and $I_{d, R M S}$ overtake the advantages from the decreased $I_{s, R M S}$ and $I_{c, R M S}$.

\section{DESIGN CONSIDERATION}

It is often the case that presenting a design guideline with a practical design example is more convenient and illustrative. The requirements for the prototype BCM TIB LED driver are assumed as $V_{i}=14 \mathrm{~V}, V_{o}=55 \mathrm{~V} \pm 15 \%$, and $I_{o}=44 \mathrm{~mA}$ for the 14 inch LED backlight of a notebook computer. The parameters to be given and to be determined are summarized in Table I.

First, the tapped-inductor turns ratio can be determined by using the conditions for the switching frequency stability and 
TABLE I

DESIGN PARAMETERS

\begin{tabular}{c|l|l}
\hline Item & \multicolumn{1}{|c|}{ Description } & \multicolumn{1}{c}{ To be } \\
\hline$A_{c}$ & Core cross-sectional area at air gap & Given: $7.1 \mathrm{~mm}^{2}$ \\
\hline$M L T_{p}$ & Primary mean length per turn & Given: $16 \mathrm{~mm}$ \\
\hline$M L T_{s}$ & Secondary mean length per turn & Given: $16 \mathrm{~mm}$ \\
\hline$\rho$ & Copper wire resistivity & Given: $1.72 \times 10^{-8} \Omega \cdot \mathrm{m}$ \\
\hline$A_{w}$ & Core window area & Given: $7.1 \mathrm{~mm}^{2}$ \\
\hline$K_{u}$ & Winding fill factor & Given: 0.6 \\
\hline$N$ & Tapped-inductor turns ratio & Determined: 1 \\
\hline$f$ & Switching frequency & Determined: $220 \mathrm{kHz}$ \\
\hline$L_{m}$ & Primary magnetizing inductance & Determined: $87 \mu \mathrm{H}$ \\
\hline$B_{p k}$ & Peak flux density & Determined: $0.16 \mathrm{~T}$ \\
\hline$N_{p}$ & Primary number of turns & Determined: 38 \\
\hline$l_{g}$ & Air gap length & Determined: $0.15 \mathrm{~mm}^{2}$ \\
\hline$A_{p}$ & Primary wire cross-sectional area & Determined: $0.082 \mathrm{~mm}^{2}$ \\
\hline$A_{s}$ & Secondary wire cross-sectional area & Determined: $0.029 \mathrm{~mm}^{2}$ \\
\hline
\end{tabular}

the ZVS of the switch. The value of $N$ for the best frequency stability at the typical output voltage of $55 \mathrm{~V}$ can be determined from (6) to be 1.93. On the other hand, to ensure the ZVS over the entire output voltage range in an actual circuit, an extra $10 \%$ margin is applied at the minimum output voltage, resulting in $N<1.01$ from (14). It should be noted that both of these conditions cannot be satisfied at the same time. Since efficiency is more critical in mobile products, the condition for the ZVS takes a higher priority. As a result, the switching frequency stability needs to be sacrificed to some extent. Therefore, the value of $N$ is finally chosen to be 1 .

It should be noted that the switching frequency range of the proposed BCM TIB LED driver to the entire output voltage range is estimated by using (5) to be $-4.2 /+3.0 \%$ of a typical value, while that of the conventional BCM boost LED driver is estimated to be $-9.2 /+11 \%$. This shows that the switching frequency stability is still significantly better in the proposed converter, even though it is somewhat sacrificed in choosing the value of $N$.

It is generally desired that the switching frequency be as high as possible to minimize the values and sizes of the reactive components. However, this is limited by a decrease in efficiency due to increases in the magnetic core loss, the switch turn-off loss, and the switch gate drive loss. Moreover, a higher frequency of operation also causes higher levels of various non-ideal side-effects such as the skin effect, proximity effects, and EMI, which are very difficult to predict quantitatively. Therefore, the switching frequency is frequently chosen on an empirical base rather than from an optimization process. In this example, the typical switching frequency at the typical output voltage condition of $V_{o}=55 \mathrm{~V}$ is chosen to be $f=220 \mathrm{kHz}$ in consideration of the aforementioned side-effects.

The magnetizing inductance of the tapped-inductor can now be obtained from (5) as $L_{m}=87 \mu \mathrm{H}$ at the typical output voltage of $55 \mathrm{~V}$. The number of turns of the primary winding $N_{p}$ and the air gap length $l_{g}$ of the tapped-inductor can be obtained from the following two equations:

$$
\begin{gathered}
L_{m}=\mu_{0} A_{c} N_{p}^{2} / l_{g}, \\
N_{p} I_{p k}=B_{p k} l_{g} / \mu_{0},
\end{gathered}
$$

where $\mu_{0}$ is the permeability of vacuum equal to $4 \pi \times 10^{-7}, A_{c}$ is the core cross-sectional area at the air gap which is given by the core manufacturer as $A_{c}=7.1 \mathrm{~mm}^{2}$, and $B_{p k}$ is the peak limit of the core flux density. Considering the large flux swing due to the BCM operation, it is a good practice to make the value of $B_{p k}$ equal to half the saturation flux density. Assuming a core made of MGB1 ferrite from TAK Technology whose saturation flux density is $0.32 \mathrm{~T}$, the value of $B_{p k}$ is chosen to be $0.16 \mathrm{~T}$. Under the maximum output voltage condition, (4), (19), and (20) can be solved to give $N_{p}=37$ and $l_{g}=0.14 \mathrm{~mm}$. According to the tapped-inductor manufacturer's standards, the value of $l_{g}$ is finally adjusted to the closest large standard value of $0.15 \mathrm{~mm}$, and the value of $N_{p}$ is adjusted to 38 to maintain the value of $L_{m}$. The number of turns of the secondary winding $N_{s}$ can be obtained from $N_{s}=N \cdot N_{p}$ as 38 .

The wire loss in the tapped-inductor can be obtained as:

$$
P_{w}=\frac{I_{i, R M S}^{2} N_{p} M L T_{p} \rho}{A_{p}}+\frac{I_{s, R M S}^{2} N_{s} M L T_{s} \rho}{A_{s}},
$$

where the definitions and values of $\rho, M L T_{p}, M L T_{s}, A_{p}$, and $A_{s}$ are listed in Table I. By using the fill factor constraint defined by:

$$
K_{u} A_{w}=N_{p} A_{p}+N_{s} A_{s},
$$

where $K_{u}$ is the winding fill factor and $A_{w}$ is the core window area, $A_{s}$ in (21) can be eliminated to give:

$$
P_{w}=\frac{I_{i, R M S}^{2} N_{p} M L T_{p} \rho}{A_{p}}+\frac{I_{s, R M S}^{2} N_{s}^{2} M L T_{s} \rho}{K_{u} A_{w}-N_{p} A_{p}} .
$$

By differentiating (23) with respect to $A_{p}$, the stationary point where the minimum of (23) occurs can be found by solving:

$$
\frac{I_{s, R M S}^{2} N_{s}^{2} M L T_{s}}{I_{i, R M S}^{2} M L T_{p}} A_{p}^{2}=\left(K_{u} A_{w}-N_{p} A_{p}\right)^{2}
$$

together with (3), (15) and (17) to give:

$$
A_{p}=\frac{K_{u} A_{w}}{N_{p}+N_{s} \sqrt{\frac{k V_{i}}{V_{o}+N V_{i}+N(N+2)\left(V_{o}-V_{i}\right)}} \sqrt{\frac{M L T_{s}}{M L T_{p}}}} .
$$




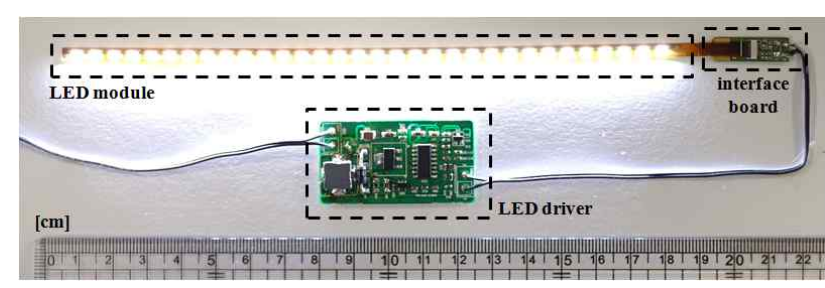

Fig. 11. Implemented setup.

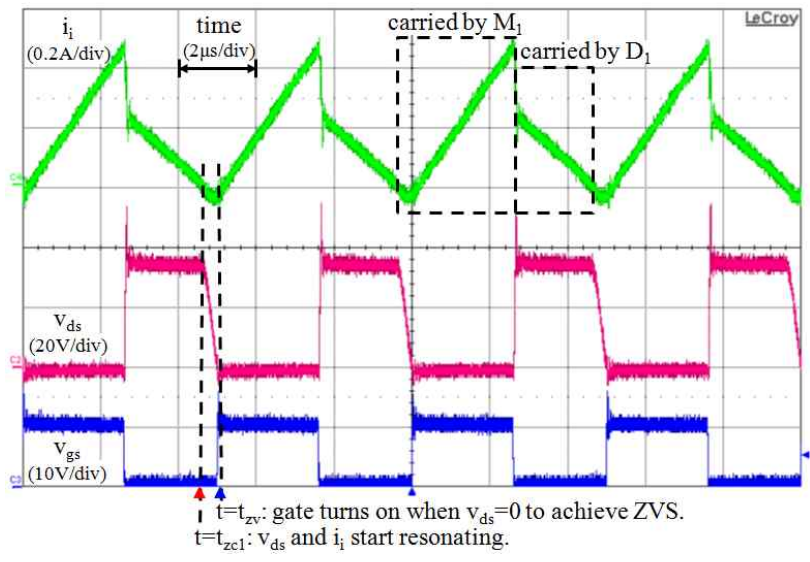

Fig. 12. Experimental waveforms.

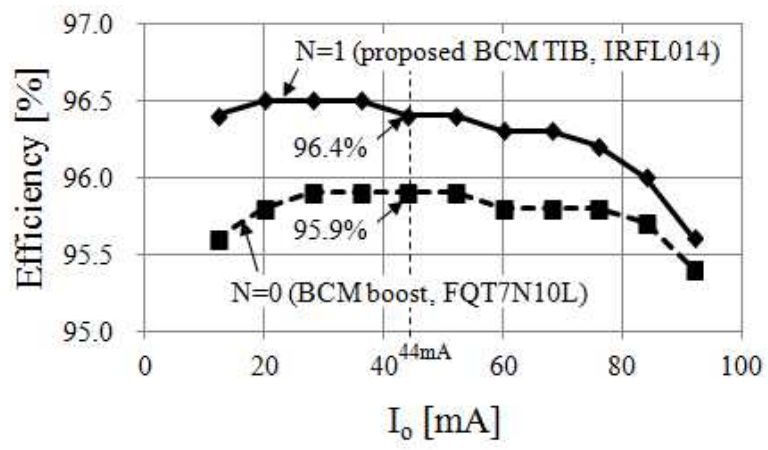

Fig. 13. Measured efficiencies.

By substituting the given and determined values listed in Table I into (25), the value of $A_{p}$ minimizing the wire loss in the tapped-inductor is obtained as $A_{p}=0.082 \mathrm{~mm}^{2}$. The value of $A_{s}$ is obtained from (22) as $A_{s}=0.029 \mathrm{~mm}^{2}$. The diameters of the primary and secondary windings are calculated to be $0.32 \mathrm{~mm}$ and $0.19 \mathrm{~mm}$ respectively. However, they are adjusted to $0.3 \mathrm{~mm}$ and $0.2 \mathrm{~mm}$ to allow for the use of standard wires.

Finally, the core loss of the tapped-inductor can be estimated from the core manufacturer's data sheet by using the switching frequency $f$ and the peak flux density $B_{p k}$. In the case of too much core loss, increasing the air gap length $l_{g}$ to reduce $B_{p k}$ is worth trying as a quick remedy. However, it should be noted that this requires more winding turns to maintain the original inductance value, resulting in an increase in the wire loss. If both the core loss and the wire loss are unacceptably high, a different core with bigger size should be considered.

\section{EXPERIMENTAL RESULTS}

A $44 \mathrm{~mA}, 14 \mathrm{~V} / 55 \mathrm{~V} \pm 15 \%$ prototype of the proposed $\mathrm{BCM}$ TIB LED backlight driver for a 14 inch notebook computer has been built as described in the previous chapter. The control circuit has been implemented with the peak current mode control scheme. The switch turns off when the switch current exceeds the output current error signal generated by an error amplifier and it turns back on when the switch voltage becomes zero to achieve ZVS. The switch current is sensed by a resistor at the MOSFET source, and the switch voltage is sensed by a resistor divider at the MOSFET drain. A 60V-rated IRFL014 with $R_{D S(O N)}=0.2 \Omega$ is chosen for the switch. A $100 \mathrm{~V}$-rated $1 \mathrm{~N} 4934$ is chosen for the diode. Since the tapped-inductor turns ratio is 1 , a bifilar winding is used to minimize the leakage inductance.

Fig. 11 shows a photo of the implemented setup. The LEDs are mounted on a flexible printed circuit (FPC). A small PCB for interfacing the FPC and wires are used in the setup. Fig. 12 shows the key experimental waveforms. Referring to Fig. 9, the moment the input current $i_{i}$ reaches zero is denoted by $t=t_{z c 1}$. The switch voltage $v_{d s}$ and the input current $i_{i}$ start resonating at $t=t_{z c 1}$, and the voltage $v_{d s}$ reaches zero at $t=t_{z v}$. The gate turns on at $t=t_{z v}$ to achieve ZVS. Due to the small tapped-inductor leakage inductance, whose measured value is $0.2 \mu \mathrm{H}$, the turn-off spike in the $v_{d s}$ waveform is small enough to avoid the use of a snubber circuit.

The switching frequency is measured to be $200 \mathrm{kHz}$, which is slightly lower than the designed value of $220 \mathrm{kHz}$. This is because the gate turn-on delay introduced to provide ZVS, which is not considered in deriving (5), reduces the switching frequency [13]. In addition to the gate turn-on delay, both the switch on-time and the switch off-time increase to maintain the averaged input current. This results in a small additional decrease in the switching frequency.

For comparison purposes, a conventional BCM boost LED driver has been made by replacing the tapped-inductor in the proposed BCM TIB LED driver prototype with an inductor using the same core. The inductor value is chosen to be $137 \mu \mathrm{H}$ to maintain the typical operating point shown in Fig. 7. The switch is also replaced with a $100 \mathrm{~V}$-rated switch since the voltage stress in the conventional driver at the maximum output voltage exceeds the maximum rating of the IRFL014. A FQT7N10L with $R_{D S(O N)}=0.35 \Omega$ is chosen since the $Q_{g} \times R_{D S(O N)}$ figure of merit (FOM) of 2.1 is very close to that of the IRFL014 at 2.2.

Fig. 13 shows the efficiencies of both of the LED drivers according to the output current $I_{o}$. At the typical output current of $44 \mathrm{~mA}$, the proposed BCM TIB LED driver shows an efficiency of $96.4 \%$, and the BCM boost LED driver shows an efficiency of $95.9 \%$. When the output current is low, the switch turn-off loss becomes dominant with a high switching frequency. When the output current is high, the conduction loss becomes dominant with a high input current. These are 

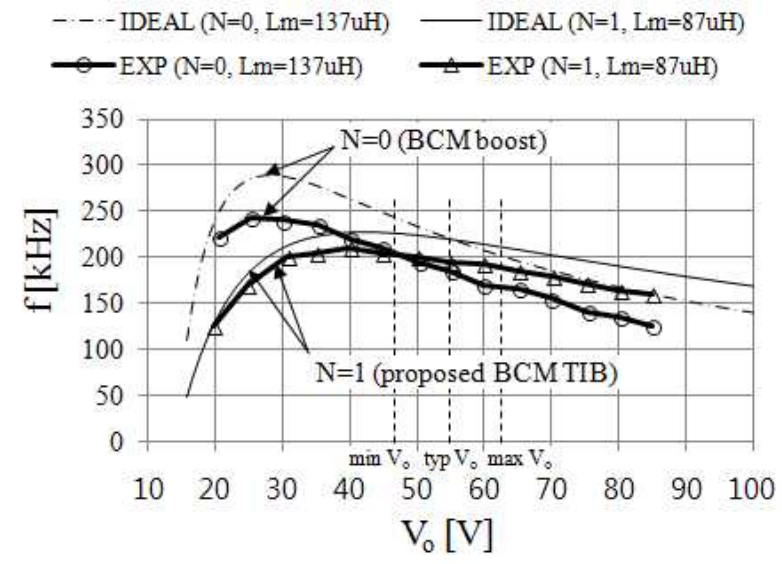

Fig. 14. Ideal and experimental switching frequencies.

reflected in Fig. 13. Since the low switch voltage stress of the proposed LED driver allows for the use of switching devices with a low voltage rating and a low $R_{D S(O N)}$, the proposed LED driver shows higher efficiency than the BCM boost LED driver.

Fig. 14 shows the ideal switching frequencies of both of the LED drivers predicted by (5) and the experimental results. It confirms that the switching frequency of the proposed LED driver is much more stable with respect to variations in the output voltage. It also shows that the gap between the ideal and the experimental curves are larger in the BCM boost LED driver. This is because the larger-valued inductance of the $\mathrm{BCM}$ boost LED driver requires a longer gate turn-on delay to achieve ZVS.

\section{CONCLUSIONS}

In low-power step-up LED driver applications where the input current is so small that the associated conduction loss is not very critical, a BCM boost converter is a good candidate since the soft-switching feature allows for an increase in the switching frequency to achieve a compact circuit design. However, the conventional BCM boost topology often fails in such applications since variations in the LED voltage causes a significant change in the switching frequency.

To overcome this problem, a BCM TIB LED driver whose switching frequency is very insensitive to changes in the output voltage is proposed. It also features soft-switching of both the switch and the diode, no diode reverse-recovery problem, a high step-up ratio, and a low switch voltage stress. Therefore, the proposed BCM TIB LED driver is considered to be very promising in small form factor mobile product applications.

\section{REFERENCES}

[1] H.-J. Chiu and S.-J. Cheng, "LED backlight driving system for large-scale LCD panels," IEEE Trans. Ind.
Electron., Vol. 54, No. 5, pp. 2751-2760, Oct. 2007.

[2] Y.-T. Hsieh, B.-D. Liu, J.-F. Wu, C.-L. Fang, H.-H. Tsai, and Y.-Z. Juang, "A high efficiency boost white LED driver for portable electronics applications," in Proc. ISNE, pp. 13-16, 2010.

[3] K.-H. Jung, J.-W. Yoo, and C.-Y. Park, "Design and implementation of a current-balancing circuit for LED security lights," Journal of Power Electronics, Vol. 12, No. 6, pp. 869-877, Nov. 2012.

[4] W. Chen and S. Y. R. Hui, "A dimmable light-emittingdiode (LED) driver with mag-amp postregulators for multistring applications," IEEE Trans. Power Electron., Vol. 26, No. 6, pp. 1714-1722, Jun. 2011.

[5] X. Qu, S.-C. Wong, and C. K. Tse, "Temperature measurement technique for stabilizing the light output of RGB LED lamps," IEEE Trans. Instrum. Meas., Vol. 59, No. 3, pp. 661-670, Mar. 2010.

[6] S. Ang and A. Oliva, Power Switching Converters, 2nd ed., CRC Press, 2005.

[7] A. I. Pressman, Switching Power Supply Design, 2nd ed. International Editions, McGraw-Hill, 1999.

[8] D. M. Sable, B. H. Cho, and R. B. Ridley, "Elimination of the positive zero in fixed frequency boost and flyback converters," in Proc. APEC, pp. 205-211, 1990.

[9] W.-C. Wu, R. M. Bass, and J. R. Yeargan, "Eliminating the effects of the right-half plane zero in fixed frequency boost converters," in Proc. PESC, pp. 362-366, 1998.

[10] X. Yang, Y. Ying, and W. Chen, "A novel interleaving control scheme for boost converters operating in critical conduction mode," Journal of Power Electronics, Vol. 10, No. 2, pp. 132-137, Mar. 2010.

[11] J.-B. Lio, M.-S. Lin, D. Y. Chen, and W.-S. Feng, "Single-switch soft-switching flyback converter," Electron. Letters, Vol. 32, No. 16, pp. 1429-1430, Aug. 1996.

[12] B.-R. Lin and C.-C. Chien, "Interleaved boost-flyback converter with boundary conduction mode for power factor correction," Journal of Power Electronics, Vol. 12, No. 5, pp. 708-714, Sep. 2012.

[13] J.-H. Park and B.-H. Cho, "The zero voltage switching (ZVS) critical conduction mode (CRM) buck converter with tapped-inductor," IEEE Trans. Power Electron., Vol. 20, No. 4, pp. 762-774, Jul. 2005.

[14] F. A. Himmelstoss and P. H. Wurm, "Low-loss converters with high step-up conversion ratio working at the border between continuous and discontinuous mode," in Proc. ICECS, pp. 734-737, 2000.

[15] J.-i. Kang, S.-K. Han, and J. Han, "Analysis and design of high-efficiency boundary conduction mode tappedinductor boost converter for LED driver applications," in Proc. KIPE Annual Fall Conference, pp. 5-6, 2013.

[16] N. Vazquez, L. Estrada, C. Hernandez, and E. Rodriguez, "The tapped-inductor boost converter," in Proc. ISIE, pp. 538-543, 2007.

[17] D.-H. Kim, J.-H. Jang, J.-H. Park, and J.-W. Kim, "Single-ended high-efficiency step-up converter using the isolated switched-capacitor cell," Journal of Power Electronics, Vol. 13, No. 5, pp. 766-778, Sep. 2013.

[18] J.-M. Kang, S.-H. Lee, S.-S. Hong, and S.-K. Han, "Voltage clamped tapped-inductor boost converter with high voltage conversion ratio," Trans. KIPE, Vol. 17, No. 1, pp. 34-40, Feb. 2012. 


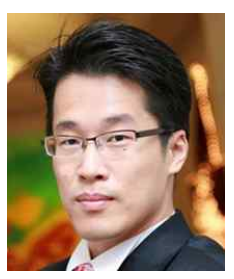

Jeong-il Kang received his B.S., M.S., and Ph.D. degrees in Electrical Engineering from the Korea Advanced Institute of Science and Technology (KAIST), Daejeon, Korea, in 1995, 1997, and 2002, respectively. In 2002, he joined Samsung Electronics Co., Ltd., Suwon, Korea, and is currently working on the design and control of semiconductor light source drivers for various display and lighting devices as a Principal Engineer in the Visual Display Research \& Development Office. His current research interests include the development, modeling, and control of power converter topologies. He is a Member of the Korean Institute of Power Electronics (KIPE).

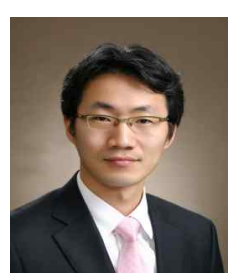

Sang-Kyoo Han received his M.S. and Ph.D. degrees in Electrical Engineering and Computer Science from the Korea Advanced Institute of Science and Technology (KAIST), Daejeon, Korea, in 2001 and 2005, respectively. For the next six months, he was a Post-Doctoral Fellow at KAIST where he developed digital display power circuits and preformed several research activities. Since 2005, he has been with the Department of Electrical Engineering, Kookmin University, Seoul, Korea, as an Associate Professor. He has also worked for the Samsung Power Electronics Center (SPEC) and the Samsung Network Power Center (SNPC) as a Research Fellow. His primary areas of research interests include power converter topologies, LED drivers, renewable energy systems, and battery chargers for electric vehicles. Dr. Han is a Member of the Korean Institute of Power Electronics (KIPE).

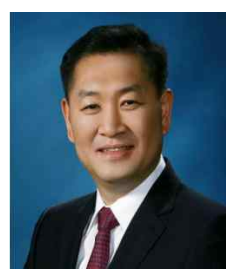

Jonghee Han is an Executive Vice President of the Visual Display Business for Samsung Electronics. In this role, he leads the Visual Display Research \& Development Office. Since joining Samsung in 1988, Mr. Han has played a significant role in the success of Samsung's Visual Display business, helping it solidify its top position within the consumer electronics industry. One of his many successes includes the launch of the award-winning 'Bordeaux' TV series, which became one of the world's best-selling LCD TVs in 2006. During his tenure, Mr. Han has accomplished a number of "firsts" in the TV industry, serving at the forefront of some of the most noteworthy TV advancements. For example, he was an integral force in the development of the world's first 3D LED TV in 2010 and first Smart TV in 2011, among numerous other developments. More recently, he played a critical role in the creation of both the world's first Curved OLED TV and Curved UHD TV. In particular, his research specialties include image processing, audio signal processing, analog/digital mixed systems, display devices, and power electronics for both innovative displays and audio products. Along with contributing his vast knowledge to Samsung's Visual Display Business, he is also a Member of the Korean Institute of Power Electronics (KIPE). Mr. Han holds a bachelor's degree in electrical engineering from Inha University, Incheon, Korea. 